\title{
Article
}

\section{Tidally induced warps of spiral galaxies in IllustrisTNG}

Semczuk, Marcin, Łokas, Ewa L, D’Onghia, Elena, Athanassoula, E, Debattista, Victor $\mathrm{P}$ and Hernquist, Lars

Available at http://clok.uclan.ac.uk/34672/

Semczuk, Marcin, Łokas, Ewa L, D'Onghia, Elena, Athanassoula, E, Debattista, Victor P ORCID: 0000-0001-7902-0116 and Hernquist, Lars (2020) Tidally induced warps of spiral galaxies in IllustrisTNG. Monthly Notices of the Royal Astronomical Society, 498 (3). pp. 3535-3548. ISSN 0035-8711

It is advisable to refer to the publisher's version if you intend to cite from the work. http://dx.doi.org/10.1093/mnras/staa2609

For more information about UCLan's research in this area go to http://www.uclan.ac.uk/researchgroups/ and search for <name of research Group>.

For information about Research generally at UCLan please go to http://www.uclan.ac.uk/research/

All outputs in CLoK are protected by Intellectual Property Rights law, including Copyright law. Copyright, IPR and Moral Rights for the works on this site are retained by the individual authors and/or other copyright owners. Terms and conditions for use of this material are defined in the policies page.

\section{CLoK}

Central Lancashire online Knowledge www.clok.uclan.ac.uk

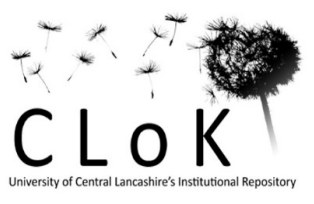




\title{
Tidally induced warps of spiral galaxies in IllustrisTNG
}

\author{
Marcin Semczuk ${ }^{\circledR},{ }^{1,2 \star}$ Ewa L. Łokas, ${ }^{2}$ Elena D’Onghia, ${ }^{3,4}$ E. Athanassoula, ${ }^{5}$ Victor P. Debattista ${ }^{\circledR 6}$ \\ and Lars Hernquist ${ }^{7}$ \\ ${ }^{1}$ Department of Physics and Astronomy, University of Leicester, Leicester LE1 7RH, UK \\ ${ }^{2}$ Nicolaus Copernicus Astronomical Center, Polish Academy of Sciences, Bartycka 18, PL-00-716 Warsaw, Poland \\ ${ }^{3}$ Department of Astronomy, University of Wisconsin, 475 North Charter Street, Madison, WI 53706, USA \\ ${ }^{4}$ Center for Computational Astrophysics, Flatiron Institute, 162 Fifth Avenue, New York, NY 10010, USA \\ ${ }^{5}$ LAM, Aix Marseille Univ, CNRS, CNES, Marseille, France \\ ${ }^{6}$ Jeremiah Horrocks Institute, University of Central Lancashire, Preston PR1 2HE, UK \\ ${ }^{7}$ Center for Astrophysics | Harvard \& Smithsonian, 60 Garden Street, Cambridge, MA 02138, USA
}

Accepted 2020 August 21. Received 2020 August 19; in original form 2020 February 17

\begin{abstract}
Warps are common features in both stellar and gaseous discs of nearby spiral galaxies with the latter usually easier to detect. Several theories have been proposed in the literature to explain their formation and prevalence, including tidal interactions with external galaxies. Observational correlations also suggest the importance of tides for warp formation. Here, we use the TNG100 run from the magnetohydrodynamical cosmological simulation suite IllustrisTNG to investigate the connection between interactions and the formation of gas warps. We find that in the sample of well-resolved gas-rich spiral galaxies $\left(10^{10} \lesssim M_{*} / \mathrm{M}_{\odot}\right.$ $\lesssim 10^{11}$ at $z=0$ ) from the simulation TNG100-1, about 16 per cent possess the characteristic S-shaped warp. Around one-third of these objects have their warps induced by interactions with other galaxies. Half of these interactions end with the perturber absorbed by the host by $z=0$. We find that warps induced by interactions survive on average for $<1$ Gyr, similarly to the remaining S-shaped warps. The angle between the orbital angular momentum of the perturber and the angular momentum of the host's disc that most likely leads to warp formation is around $45^{\circ}$. While our main goal is to investigate tidally induced warps, we find that during interactions in addition to tides, new gas that is accreted from infalling satellites also can contribute to warp formation.
\end{abstract}

Key words: galaxies: evolution - galaxies: interactions - galaxies: kinematics and dynamics - galaxies: spiral - galaxies: structure.

\section{INTRODUCTION}

Galactic warps are vertical distortions of stellar and gaseous discs of spiral galaxies. Observationally, warps are found in a large fraction of nearby edge-on, or close to edge-on, galaxies (Bosma 1991; GarcíaRuiz, Sancisi \& Kuijken 2002). They are more prominent in H I observations since usually they appear at radii where stellar discs are already fading (Briggs 1990). The most common type of warp has one side of the disc bent upwards and the other downwards. This kind of warp is often referred to as 'integral sign' or S-shaped. All three massive spirals of the Local Group have S-shaped warps. The gas warp of the Milky Way (MW) was the first discovered warp back in the 1950s (Burke 1957; Kerr 1957), while the warps of M33 and M31 were detected two decades later (Rogstad, Wright \& Lockhart 1976; Newton \& Emerson 1977). Recent results from the stellar surveys of the MW shed more light and attention on the subtle stellar warp of our Galaxy (e.g. Poggio et al. 2018, 2020; Schönrich \& Dehnen 2018; Chen et al. 2019; Romero-Gómez et al. 2019; Skowron et al. 2019).

Several theories have been proposed in the literature trying to explain the formation and frequency of galactic warps. They relate

^E-mail: mbs19@leicester.ac.uk the formation of warps to the misalignment between the angular momentum of the halo and the disc (Debattista \& Sellwood 1999), the cosmic accretion of the material on to the galactic disc (e.g. Shen \& Sellwood 2006), the misalignment between the inner disc and the hot gas halo that leads to the asymmetric accretion (Roškar et al. 2010), the interaction between the gaseous disc and extragalactic magnetic fields (Battaner, Florido \& Sanchez-Saavedra 1990; Battaner \& Jimenez-Vicente 1998), or the gravitational coupling between the spiral arms and the warp (Masset \& Tagger 1997). In this paper, we investigate a scenario that was also proposed earlier, i.e. that warps originate from tidal interactions with external galaxies, as occur naturally within the framework of cosmological simulations.

It was demonstrated in various numerical simulations that a flyingby perturber can tidally induce vertical distortions in discs of host galaxies. The condition for such a scenario to occur is that the orbit of the perturber is inclined with respect to the plane of the host's disc. The perturber can be either a less massive satellite on a tight orbit (D'Onghia et al. 2016) or a more massive galaxy on a wide orbit (Vesperini \& Weinberg 2000; Kim et al. 2014). Cosmological simulations of MW-like galaxies show that not only interactions with satellites can create vertical distortions due to tidal forces (Gómez et al. 2016) but also the impact of the satellite can be amplified by the dark halo wake (Gómez et al. 2017), which can generate additional corrugation patterns in the stellar disc. Semczuk et al. (2018) showed 
that M33 could have had its stellar and gaseous warps induced by a recent interaction with $\mathrm{M} 31$, where the gas warp was shaped not only by the tidal field, but also by the mild effect of ram pressure from the hot gas halo of M31. Kim et al. (2014) used idealized simulations to study the process of stellar warp formation via tidal interactions. Interestingly, these authors argue that the parameter that strongly affects the strength and longevity of warps is the incident angle between the angular momenta of the perturber on its orbit and the stars in the host's disc. They found that the strongest and most persistent warps are formed when this angle takes values of $45^{\circ}$ and $135^{\circ}$, which optimizes the integration time of the interaction with the vertical component of the tidal force.

A few observational studies also indicate a connection between warps and tidal interactions. Kollatschny \& Dietrich (1990) argued that the very strong stellar warp of Mkn 306 originated from the interaction with the close-by Mkn 305. The proximity of the perturber and the lack of gas argue against other explanations (e.g. gas accretion or interaction of gas with magnetic fields) of this warp's formation. Using a sample of 540 optical images of galaxies, Reshetnikov \& Combes (1998) found that the frequency of warps is higher in galaxies having a companion or interacting than in isolated objects. Schwarzkopf \& Dettmar (2001) discovered that stellar warps are on average 2.4 times bigger in interacting galaxies than in isolated ones. Ann \& Park (2006) and García-Ruiz et al. (2002) showed that stellar and gas warps are more asymmetric when they are associated with interactions or higher density environments, respectively. This correlation can possibly be used as a diagnostic for the formation scenario of a given warp.

In this paper, we use a simulated universe of galaxies from IllustrisTNG (Marinacci et al. 2018; Naiman et al. 2018; Nelson et al. 2018, 2019b; Pillepich et al. 2018b, 2019; Springel et al. 2018) to check whether these simulations reproduce interesting warp features of disc morphology and to investigate how important tidal interactions are in their formation. Cosmological simulations like IllustrisTNG that take into account baryonic physics, while having several shortcomings and difficulties of their own, are often successful in reproducing the observed Universe and galaxies within; therefore, they can be useful in understanding what happens in the observed Universe. In Section 2, we briefly describe the simulations we used and the sample of warped galaxies we selected from all galaxies in the simulation. In Section 3, we discuss the properties of a subtype of warped galaxies, i.e. those with S-shaped warps, and we present our results on how important tidal interactions are in shaping them. Section 4 contains the discussion and conclusions of this paper.

\section{THE SAMPLE OF SIMULATED SPIRAL GALAXIES}

\subsection{The IllustrisTNG simulation}

To investigate warping of galactic discs, we used the TNG100 simulation of the IllustrisTNG project (Marinacci et al. 2018; Naiman et al. 2018; Nelson et al. 2018; Pillepich et al. 2018b; Springel et al. 2018), which follows the formation and evolution of galaxies within the Lambda cold dark matter cosmological paradigm. IllustrisTNG is a continuation of the original Illustris project (Genel et al. 2014; Vogelsberger et al. 2014a, b) and uses the hydrodynamical movingmesh code AREPO (Springel 2010). The code was modified for the purpose of the IllustrisTNG simulations to take into account various physical phenomena relevant to the galaxy formation. These phenomena included radiative gas cooling modulated by a timedependent ultraviolet background, star formation via a subgrid model as described in Springel \& Hernquist (2003), galactic winds coming from metal enriching supernovae (Pillepich et al. 2018a), two distinct channels of black hole feedback (Weinberger et al. 2017), and growing black holes fed by accretion. Cosmological parameters used in the simulation were adopted from the Planck mission results (Planck Collaboration XIII 2016). In addition to the models of galactic and extragalactic physics, magnetic fields (Pakmor \& Springel 2013) were also coupled to the hydrodynamics. The TNG100 data of the IllustrisTNG project are publicly available (Nelson et al. 2019a).

IllustrisTNG provides an improvement in the context of galactic morphology in comparison to the original Illustris. Nelson et al. (2018) showed that mock optical colours of simulated galaxies qualitatively resemble well the Sloan Digital Sky Survey (SDSS) colours of galaxies. Rodriguez-Gomez et al. (2019) compared synthetic images of IllustrisTNG galaxies with Pan-STARSS observations and found a good agreement in terms of non-parametric morphological diagnostics, e.g. Gini- $M_{20}$ diagram. Peschken \& Lokas (2019) and Rosas-Guevara et al. (2020) used, respectively, Illustris and IllustrisTNG simulations to investigate bar formation in disc galaxies and both found the increase in the bar fraction with stellar mass that is in agreement with observations. Yun et al. (2019) used the box of $100 \mathrm{Mpc}$ from the IllustrisTNG suite to investigate the so-called jellyfish galaxies that have their morphologies shaped by the ram pressure stripping (RPS) in the hot gas haloes. They found a plethora of stripped galaxies and carefully investigated their properties and dependences on the relative orbits and positions within clusters, which showed that IllustrisTNG has enough resolution to track the evolution of gas morphologies of galaxies. Pop et al. (2017, 2018) showed that the shells commonly seen in massive elliptical galaxies naturally arise in the simulations from major mergers. Based on TNG100, Zhu et al. (2018) argued that the low surface brightness disc of Malin-1 may have originated from an interaction with a binary companion. Recently, Blumenthal et al. (2020) analysed pairs of interacting galaxies in IllustrisTNG, and demonstrated that signatures of galaxy-galaxy interactions are also present in the simulation.

\subsection{Sample selection}

In observations, gas warps are found to be more pronounced and easier to detect than stellar warps. This is probably because gas discs are less bound than stellar ones and they interact with the environment via more channels than only through gravity. For these reasons, we decided to focus our study on gas warps. To select galaxies with warped gas discs from IllustrisTNG, we classified by eye edgeon morphologies of gaseous galactic discs. This classification is described in the next section. We chose the simulation TNG1001 because the box of $100 \mathrm{Mpc}$ length was the one with the highest resolution available at the time this project was started and high resolution is crucial to investigating galactic morphologies. In order to reduce the number of galaxies for visual classification, we narrowed our sample to galaxies that at redshift $z=0$ have discy shape, contain enough gas material, and have sufficiently high resolution to reproduce morphological features. These requirements were met by applying the following selection criteria:

(i) the number of stellar particles $>10^{4}$;

(ii) the number of gas cells $>10^{4}$;

(iii) the fractional mass of stars behaving kinematically as a disc (i.e. with circularity $\epsilon>0.7$ ), $f_{\epsilon}>0.2$ (following Peschken \& Łokas 2019); 
(iv) the flatness of stellar distribution, defined as $\gamma=$ $M_{1} / \sqrt{M_{2} M_{3}}$, where $M_{1}<M_{2}, M_{3}$ are eigenvalues of the stellar mass tensor, $\gamma<0.7$ (Peschken \& Łokas 2019);

(v) the gas mass fraction $M_{\text {gas }} / M_{\text {stars }}>0.01$ within twice the halfmass stellar radius (following Yun et al. 2019);

(vi) no peculiarities in the face-on gas distribution (e.g. big holes), judged by visual inspection.

Applying all the above criteria yielded a sample of 1593 discy gasrich galaxies with enough resolution to analyse their morphology. We refer to these galaxies as our default sample. The stellar mass range of this sample is $10<\log \left(M_{*} / \mathrm{M}_{\odot}\right)<11.89$ with the median being $10^{10.33} \mathrm{M}_{\odot}$. Selected galaxies represent 24.6 per cent of all galaxies in a given mass range.

\subsection{Morphological classification}

We generated images of the edge-on distributions of neutral hydrogen in six equally spaced orientations for the galaxies of the default sample to visually classify their morphology. The neutral hydrogen fractions were given in snapshots of the simulations and were calculated by including photoionizing rate from the ultraviolet background of Faucher-Giguère et al. (2009) that accounts for selfshielding of ionizing photons (Rahmati et al. 2013). These fractions neglect molecular hydrogen, which is mostly relevant for the centres of galaxies, but not the outer parts of discs. We have done the visual classification twice to reduce human bias. Similarly to observational studies (e.g. Kuijken \& Garcia-Ruiz 2001), we divide edge-on gas morphologies into four different types:

(i) S-shaped warps (also referred to in the literature as 'integral sign' warps);

(ii) flat discs;

(iii) RPS-like morphologies (having clear features of a jellyfish galaxy, e.g. tails);

(iv) irregular warps (not having any of the above three characteristics).

Examples of galaxies of each type are presented in Fig. 1. S-shaped galaxies were qualified by the condition to have the characteristic shape present in at least one of the six projections. We note that all images were generated in square fields of side length equal to 12 times the stellar half-mass radius. Such a size was chosen to focus more on the characteristic morphology of the discs rather than on external structures. This was motivated by the plan to focus our analysis on $\mathrm{S}$-shaped warps, indicative of tidal interactions. However, the small size of the fields could lead to omission of some extended features like tails or streams and therefore we could miss some of the RPSlike objects. We refer to the work of Yun et al. (2019) who already extensively analysed jellyfish galaxies of TNG100 simulations and in this paper we narrow our focus to S-shaped warps.

Table 1 presents the results of the morphological classification in terms of relative abundances of the different types. Roughly half of all gas discs turned out to be vertically disturbed with the other half approximately flat. This is in agreement with the seminal observational finding called first Bosma's law (Bosma 1991; GarcíaRuiz et al. 2002) stating that at least half of the H I gas discs are warped. Among the whole sample, the RPS-like galaxies seem to be underrepresented. In comparison, Yun et al. (2019) found 196 jellyfish galaxies at $z=0$, which is 5.6 times more than in this study. This discrepancy arises due to many differences between the classification processes done here and by Yun et al. (2019), from which the most significant seem to be the cut in numbers of particles.
Their 3000 stellar particles extend the lower bound of the stellar mass to $10^{9.5} \mathrm{M}_{\odot}$, which gives much more objects that have the highest 'jellyfish fraction' $(\sim 0.5)$.

We checked that the fact that galaxies belong to the specific morphological warp type does not correlate with global parameters of these galaxies, e.g. total mass, half-mass radius, or gas fraction.

\section{CHARACTERISTICS OF S-SHAPED WARPS}

\subsection{Warp parametrization}

To find out what processes are most often responsible for warp formation, we need to know how warps are evolving. In order to characterize warp evolution, we decided to use the so-called warp equation (as discussed in Chen et al. 2019)

$z(R)=a_{\mathrm{w}}\left(R-R_{\mathrm{w}}\right)^{b} \sin \left(\phi-\phi_{\mathrm{w}}\right)$,

where $z$ and $R$ are cylindrical coordinates, and $a_{\mathrm{w}}, R_{\mathrm{w}}, \phi_{\mathrm{w}}$, and $b$ are warp parameters characterizing, respectively, its inclination, onset radius, line of nodes, and shape. For simplicity, we will assume the linear approximation for warps, which means that $b \equiv 1$. The parameter that is of most interest for us is $a_{\mathrm{W}}$ as it describes how strongly the disc is vertically warped. To find $a_{\mathrm{W}}$ for a given gas disc at a given snapshot, we first level the galaxy by aligning its stellar angular momentum with the $z$-axis of the coordinate system and later we divide the gas cells into eight equally spaced slices in cylindrical azimuth. The result of this division is that some slices contain parts of the disc that are flatter and some of them have the tips of the warp. To each slice, we fit the reduced equation (1), i.e.

$z(R)=a_{\mathrm{w}}\left(R-R_{\mathrm{w}}\right)$.

The factor $\sin \left(\phi-\phi_{\mathrm{w}}\right)$ disappeared as slicing the disc into parts is equivalent to deriving the azimuthal dependence. An example of the fit is presented in Fig. 2. To optimally measure the warp's vertical distortion, we average only the two maximal values of $\left|a_{\mathrm{w}}\right|$ to include the parts with the tips of the warp on both sides. In observational studies (e.g. Chen et al. 2019; Skowron et al. 2019), equation (1) is often fitted only to the region $R>R_{\mathrm{w}}$, while for $R<R_{\mathrm{w}}$ a flat line is adopted. While this two-component fit better reflects the warp geometry, it is technically more difficult to apply to a sample of thousands from cosmological simulations. We discuss these difficulties and justify the application of a simple onecomponent fit used here in Appendix A.

The method of quantifying warps described above is dependent on and sensitive to two parameters: $R_{\text {cut }}$ and $z_{\text {cut }}$, the cuts in cylindrical coordinates from which the gas cells are assigned to the given galaxy. Taking these values too big may result in including some cells that are part of an external gas filament and can result in a warp fitted badly. Taking these cuts too small may end up in retaining only the inner flat part of the disc and obtaining $\left|a_{\mathrm{w}}\right|$ too small, perhaps not even detecting the warp. In order to find the optimal values of $R_{\text {cut }}$ and $z_{\text {cut }}$, we used visually classified samples of flat discs and S-shaped warps and for $z=0$ fitted equation (2) on a grid of values of these two parameters. The goal of this procedure was to find the best values that would separate these two morphological types. We found that cuts equal to $R_{\text {cut }}=5 R_{1 / 2 *}$ and $z_{\text {cut }}=2 R_{1 / 2 *}$, where $R_{1 / 2 *}$ is the stellar half-mass radius, separated best the distributions of $a_{\mathrm{w}}$ for these two different types (see Fig. 3, where colour lines represent visually classified samples and their correspondence to the defined parameter).

Histograms for flat discs and S-shaped warps in Fig. 3 have a significant overlap, which means that for some values the parameter 

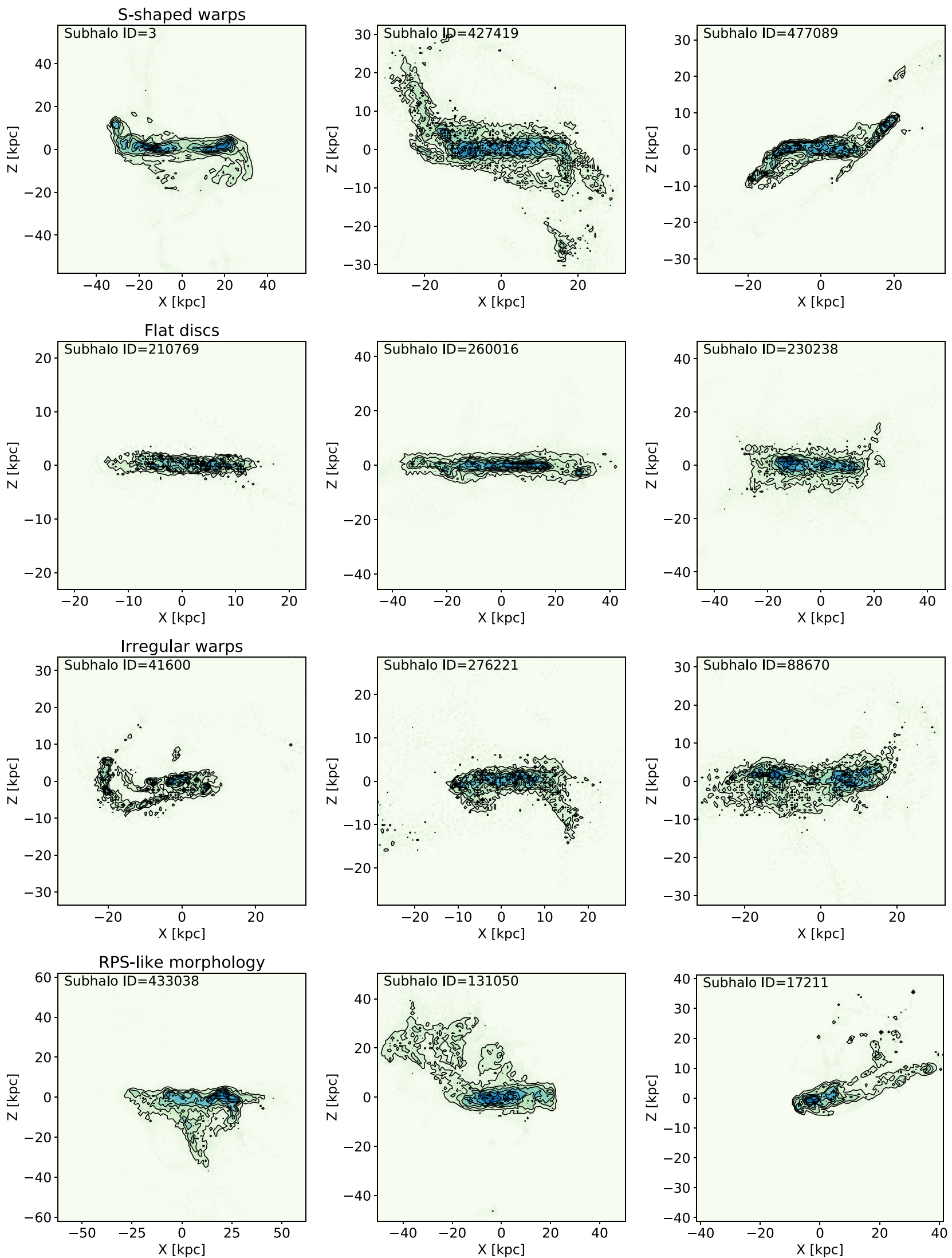

Figure 1. Neutral hydrogen edge-on distributions for four different morphological types of discs considered in this paper. Each of the four rows presents three examples of a given morphology, namely (from top to bottom) S-shaped warps, flat discs, irregular warps, and the galaxies with RPS-like morphologies. The colour scale was set to be linear in gas density to highlight better the morphological features. 
Table 1. Results of the visual morphological classification.

\begin{tabular}{lcc}
\hline Type & Number of galaxies & Percentage \\
\hline S-shaped warps & 249 & 15.6 \\
Flat discs & 855 & 53.7 \\
Irregular warps & 454 & 28.5 \\
RPS-like morphologies & 35 & 2.2 \\
\hline
\end{tabular}

$a_{\mathrm{w}}$ is not uniquely classifying the galaxy as one or the other type. In order to make sure that we analyse the clearest S-shaped warps, we reduced our sample by selecting only those galaxies for which $a_{\mathrm{w}}>0.21$, where the threshold value was chosen as the one at the intersection of the two normalized histograms. This reduction limited the number of S-shaped warped galaxies to 187 . We will refer to this sample of galaxies as our reduced S-shaped sample.

\subsection{Evolution of tidal warps}

To check whether the warp of a given galaxy was tidally induced, we looked for a relatively massive subhalo appearing in the proximity of its host galaxy in the past 5 Gyr. Once the suspect for the perturber is identified, we compare its orbit and pericentre passages with the evolution of $a_{\mathrm{w}}$. We looked whether $\pm 0.5 \mathrm{Gyr}$ around each of the pericentre passages there was a rapid increase of $a_{\mathrm{w}}$. The rapid increases were found by finding maxima of the time derivative of smoothed $a_{\mathrm{w}}$ and times where the threshold of 0.21 was crossed (for most recent warps). These coincidences could give many false results, as $a_{\mathrm{w}}$ could increase due to some one-sided structure in the past of the gas disc. Because of that, each case with a coincidence was carefully inspected by eye by tracking positions of the perturber and the edge-on morphology of the host at the corresponding time. Additionally to this visual inspection, we calculated mass ratios and a parameter quantifying tidal force to be sure that the observed tidal

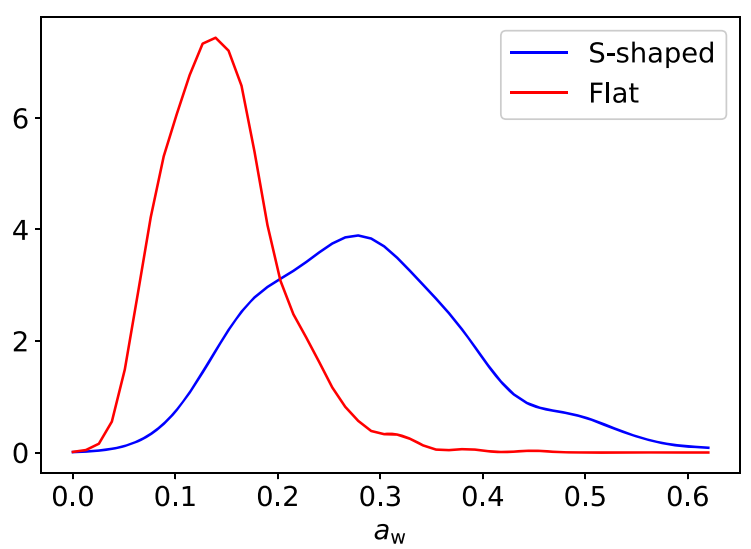

Figure 3. Normalized histograms of $a_{\mathrm{w}}$ for visually classified S-shaped warped galaxies and flat disc galaxies calculated at $z=0$ with optimal cuts $R_{\text {cut }}=5 R_{1 / 2 *}$ and $z_{\text {cut }}=2 R_{1 / 2 *}$.

perturbation was strong enough, and the warp was probably not caused by some other source (e.g. misalignment of angular momenta of the disc and the halo) that would not be visible in this visualization. To quantify the tidal force, we use the parameter $P$ (as discussed in Oh, Kim \& Lee 2015; Semczuk et al. 2018):

$P=\left(\frac{M_{\text {pert }}}{M_{\text {host }}}\right)\left(\frac{R_{\text {host }}}{d_{\text {peri }}}\right)^{3}$,

where $M_{\text {pert }}$ are the masses of perturbers measured at the last apocentre before the encounter, $M_{\text {host }}$ are the masses of host galaxies measured at the pericentre within pericentric distances $d_{\text {peri }}$, and $R_{\text {host }}$ are linear sizes of hosts defined here to be $5 R_{1 / 2 *} . P$ measures the tidal force exerted on the host and it was shown that the disc can
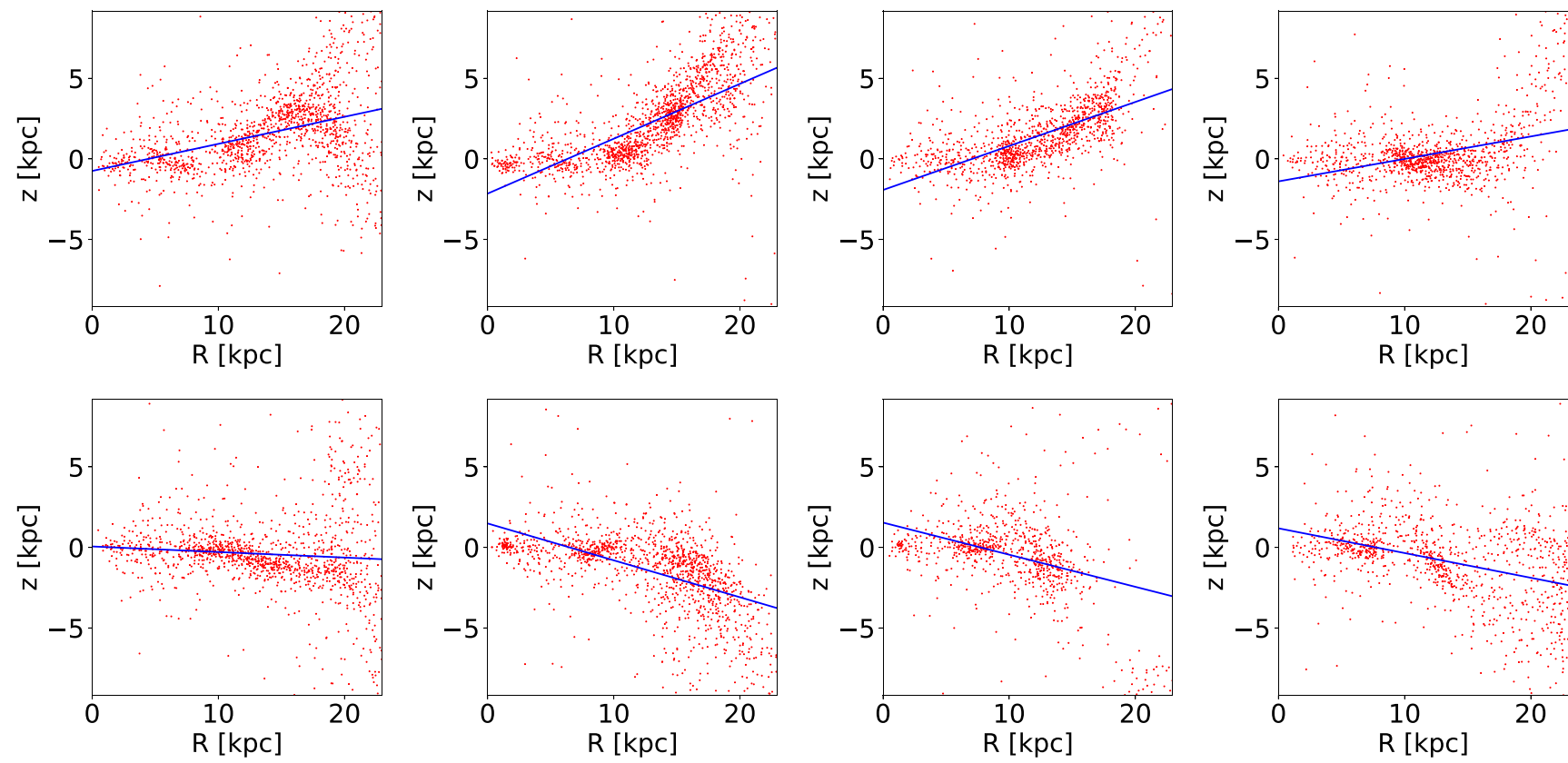

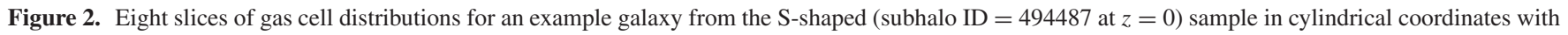

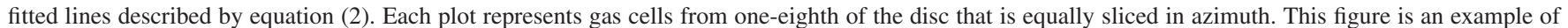
the procedure described in Section 3.1. 

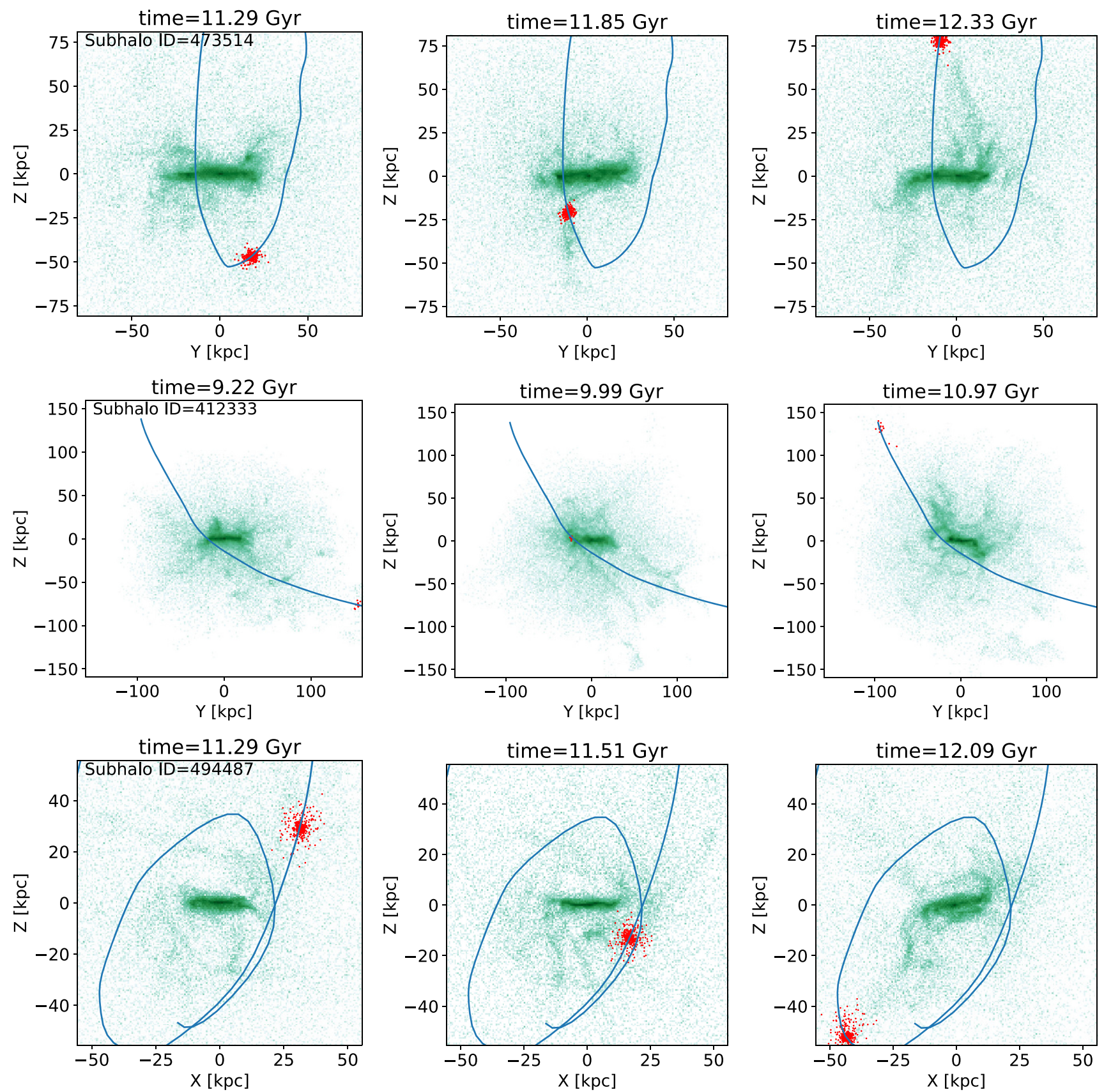

Figure 4. Examples of tidal interactions between gaseous discs of galaxies and flying-by perturbers. Each row represents a particular galaxy and each column a different snapshot. Middle column snapshots are snapshots closest to the pericentre passage, while the left ones present images before the pericentre and the right ones after that. Red points are stellar particles assigned to the perturber and blue lines are perturber orbits obtained by second-degree spline interpolation. For each case, the subhalo ID given is that of $z=0$. Unlike Fig. 1, here the colour scaling of gas density is logarithmic to enhance the surrounding medium.

respond to $P$ as low as 0.01 (Byrd \& Howard 1992) in terms of spiral arm formation.

Applying the procedures described above yielded that 66 out of 187 ( 35 per cent) warped galaxies from the reduced S-shaped sample had their warps induced by interactions with other galaxies (at some point in the last $5 \mathrm{Gyr}$ ). We note that 41 out of these 66 warps either survived until $z=0$ or were recurrently induced by the same perturber until $z=0$. From all 66 cases, half of the encounters were caused by the flying-by galaxy or a satellite and the other half were caused by satellites that ended up being accreted by the host (i.e. their identity in the Subfind catalogues was lost and passed to the host galaxy). We note that out of all of these interaction cases it is difficult to discriminate whether the formation of the warps was due entirely to the tidal force acting vertically on discs or it was an effect of the perturber collecting the circumgalactic gas and dropping it at the host's disc causing non-planar perturbations resulting in Sshaped warps. We suspect that for some cases the origin of warps could be due to the combined effect of the two phenomena.

Six examples of tidally induced warps are presented in Figs 4-7. Figs 4 and 5 present three snapshots of flying-by perturbers on their 

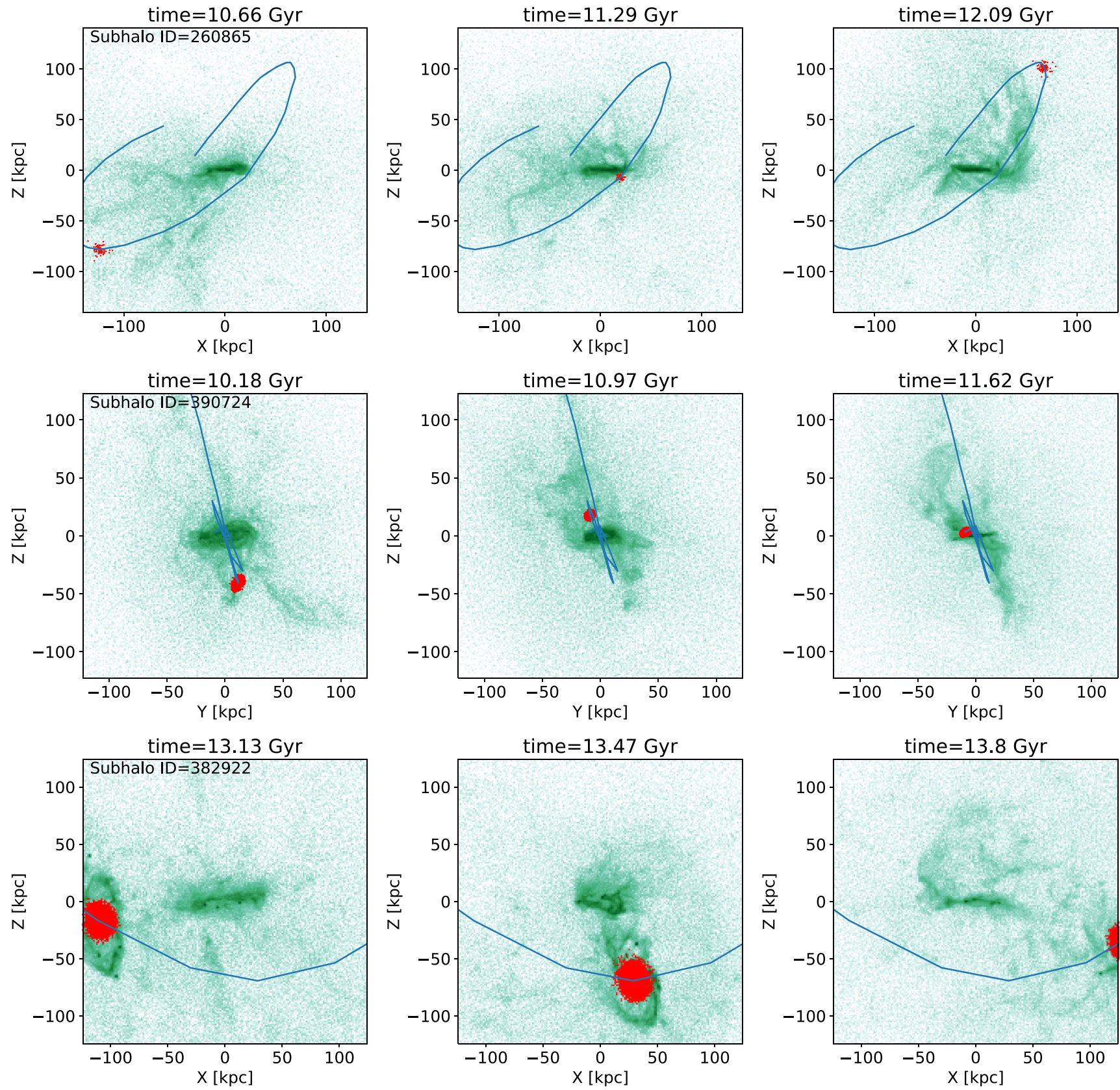

Figure 5. Same as Fig. 4, but for three other galaxies.

orbits around hosts and the reactions of the hosts' gaseous discs. The mass ratios between the perturbers and hosts are, respectively, 2.2, 3, and 3.6 percent for Figs 4 and 6 from top to bottom, and 6, 23, and 859 percent for Figs 5 and 7 from top to bottom. In the first three cases and the last one, the scenario is very similar. Before and during the pericentre, the gas disc is more or less flat, with some irregular structures. Shortly after the pericentre, the edgeon profile is transformed into the characteristic S-shape. The tidal forces originating from a perturber that is passing by on an orbital plane that is not parallel to the equatorial plane of the gaseous disc were able to induce these symmetrical morphological features. The whole process is further illustrated in Figs 6 and 7, where the values of $a_{\mathrm{w}}$ tend to rapidly increase soon after pericentre. Gaseous discs in these cases are not perfectly flat prior to the tidal perturbation, as constant accretion in cosmological simulations rarely forms very regular discs. This is also reflected in Fig. 6, where $a_{\mathrm{w}}$ has values close to $0.15-0.2$ prior to the pericentre (especially in the last example). However, the passage of the perturber increases these values to make them sometimes twice as high as the average before the event.

For the fourth and fifth examples, the scenario seems to be a bit altered. For the fourth example, we see that the gas that was initially below the host's disc seems to be partially dragged upwards by the perturber. In the end, the gas that remained below the disc, together with the part that was dragged in, contributes to the asymmetric Sshape. In the fifth case that ends in the accretion of the perturber, non-planar gas seems to be both dragged by the perturber and accreted on to the host from above and below, which results in the S-shaped morphology. These two cases illustrate well the difficulty 

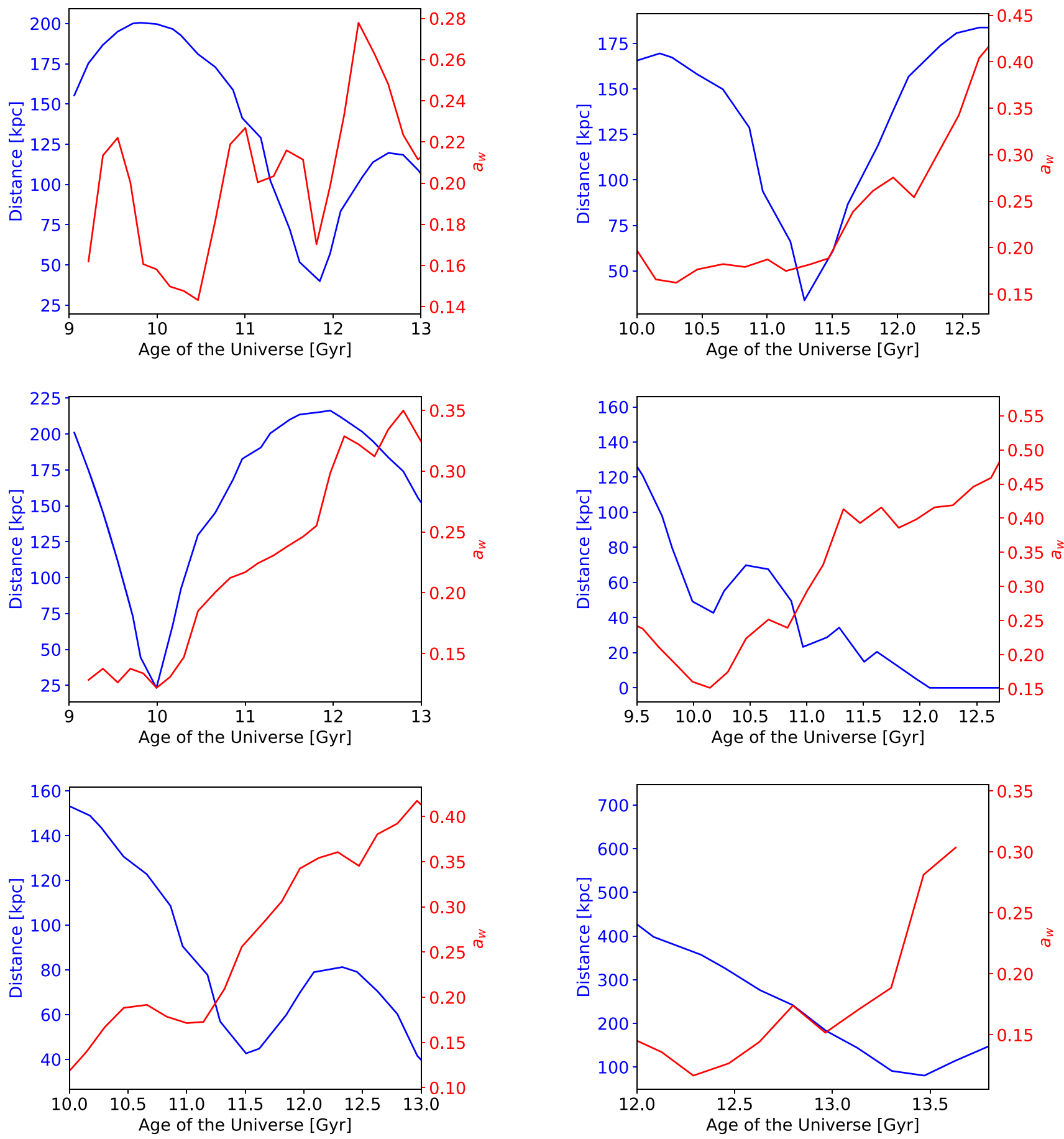

Figure 6. Time dependence of distances between host galaxies and perturbers (blue lines) for the three examples presented in Fig. 4 compared with evolutions of the warp parameter $a_{\mathrm{w}}$ measured for the gas discs of the hosts (red lines)

of separating tidal effects from the accretion of the gas caused by the satellite. While their tidal parameters seem to be very high $(P=0.17$ and 2.43), the analysis of the gas maps shows that the satellites may play important roles in mixing and accreting the surrounding gas of the host. We test this interpretation further in Section 3.6.

Fig. 8 shows histograms of pericentric distances of the perturbers, mass ratios between perturbers and hosts, masses of perturbers, and

Figure 7. Same as Fig. 6, but for three other galaxies whose encounter is shown in Fig. 5.

tidal parameters $P$ for the tidally induced warped galaxies divided into cases that did or did not end up in accretion. The accretion sample naturally has lower pericentres as for the other sample they have to be large enough for the perturber to not be dragged and accreted by the host. Smaller pericentres for the accretion sample result in higher mass ratios, since masses of the hosts are measured inside the pericentric distances. A similar distribution of perturber masses seems to confirm that. Both higher mass ratios and small 

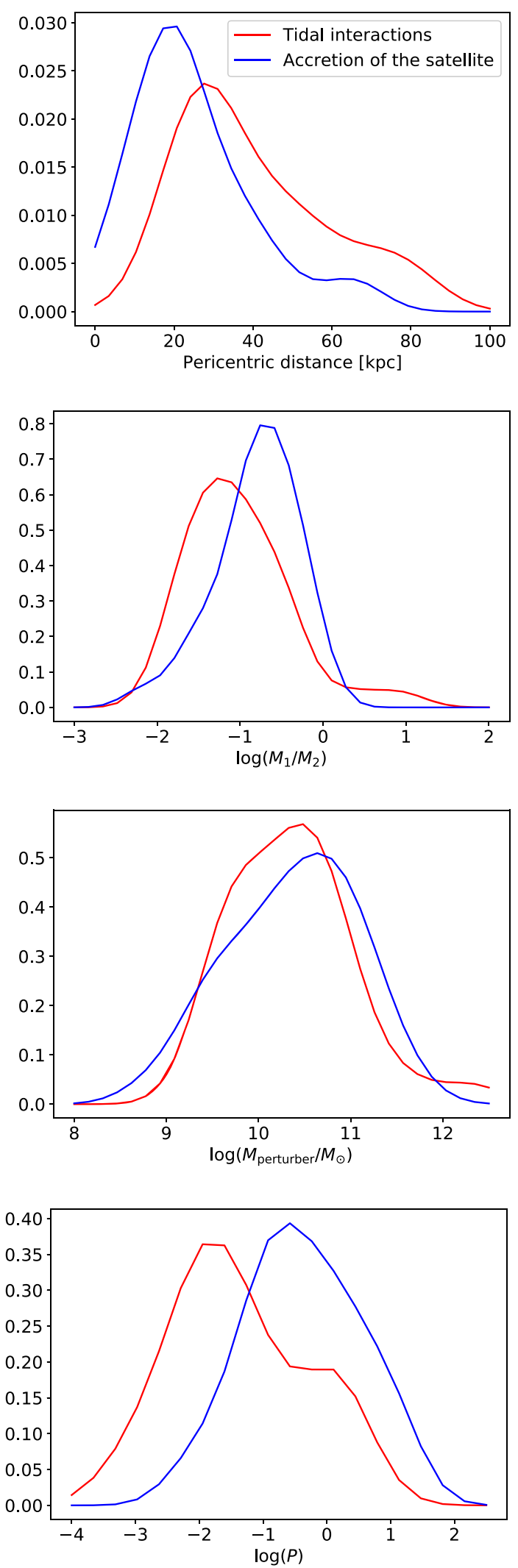

Figure 8. Normalized histograms of pericentric distances (first panel), (perturber to host) mass ratios (second panel), masses of the perturbers (third panel), and tidal parameters (last panel) for the warped galaxies that experienced tidal interaction with a flying-by perturber (red lines) and those that accreted their satellite (blue lines). pericentres contribute to higher values of $P$ for the accretion sample, which means that these encounters are more violent than the cases where the perturber flies away and does not interact with the host so tightly.

\subsection{Lifetimes of tidally induced warps}

To find the rapid increase of $a_{\mathrm{w}}$ that was used to determine whether the warp formation was caused by a flying-by perturber, we looked for a maximum of the derivative of the smoothed time evolution of $a_{\mathrm{w}}$ (or for a moment when a threshold was exceeded). This helped us to establish when warps were created. We used a similar approach to find out when warps start to dissolve by looking for a minimum in the derivative of the smoothed time evolution of $a_{\mathrm{w}}$. We defined the time between the maximum of smoothed $\mathrm{d} a_{\mathrm{w}} / \mathrm{d} t$ associated with a pericentre passage of a perturber and the following minimum as a lifetime of the tidally induced warp $\tau_{\mathrm{w}}$. Obtaining this kind of approximate value was possible for 51 out of 66 cases that we found to be driven by interactions. For the remaining 15 cases, it was impossible to achieve because the simulation ended before the minimum of the derivative occurred, i.e. the warp was induced very recently. For our sample, we found an average value of $\tau_{\mathrm{w}}=$ $0.8 \pm 0.5$ Gyr. We did a similar exercise for remaining S-shaped warps to compare their lifetimes. While the source of their warps is still to be found, we picked minimum and maximum of smoothed $\mathrm{d} a_{\mathrm{w}} / \mathrm{d} t$ around the last peak of $a_{\mathrm{w}}$. This yielded a very similar result of $0.8 \pm 0.3 \mathrm{Gyr}$, showing that lifetimes of warps are not a function of their origin. The numerical definition of $\tau_{\mathrm{w}}$ does not necessarily mean that after that time the warp is dissolved since the minimum of the derivative means only that it starts to decrease, but not necessarily disappear. Given that, it is fair to round up that number and say that the average lifetime is $\sim 1$ Gyr. This number is not an outlier in comparison with the values for stellar warps from Kim et al. (2014). They derived lifetimes even up to 4-5 Gyr, but these were obtained in idealized simulations tailored to produce strong warps, unlike the more realistic cosmological set-up used in this paper.

\subsection{Incident angles of interactions that result in warped discs}

The angle between the angular momentum of a perturber on its orbit and the angular momentum of a rotating disc was often discussed in the literature as an impactful factor on tidally induced morphologies (e.g. D’Onghia et al. 2010; Łokas et al. 2015). The incident angle (also referred to as the inclination angle) $i$ was found to have the biggest impact on galaxies when the perturber orbits were prograde $\left(i=0^{\circ}\right)$ and the smallest when they were retrograde $\left(i=180^{\circ}\right)$. For these values, however, no warp would be induced as there is little or no tidal force acting in the vertical direction on the host's disc.

We calculated incident angles for our sample of interaction-driven warps in the snapshots closest to the pericentre and plotted the normalized histogram of their cosines in the upper panel of Fig. 9. From the analysis of the histogram, one can see that most warps were induced with $i \simeq 50^{\circ}$ and in addition a small increase departing from the overall distribution can be found at $i \simeq 125^{\circ}$. This finding means that most warps were induced in encounters with incident angles close to these two values (with much greater importance of the first one). This can be interpreted as the fact that for these encounters warps were strong enough and lived long enough to be easily detectable, with the case lying close to the prograde orientation having much bigger significance. This finding is consistent with the results of Kim et al. (2014) who found that the strongest and most long-lived warps are created for angles of $45^{\circ}$ and $135^{\circ}$. Their 

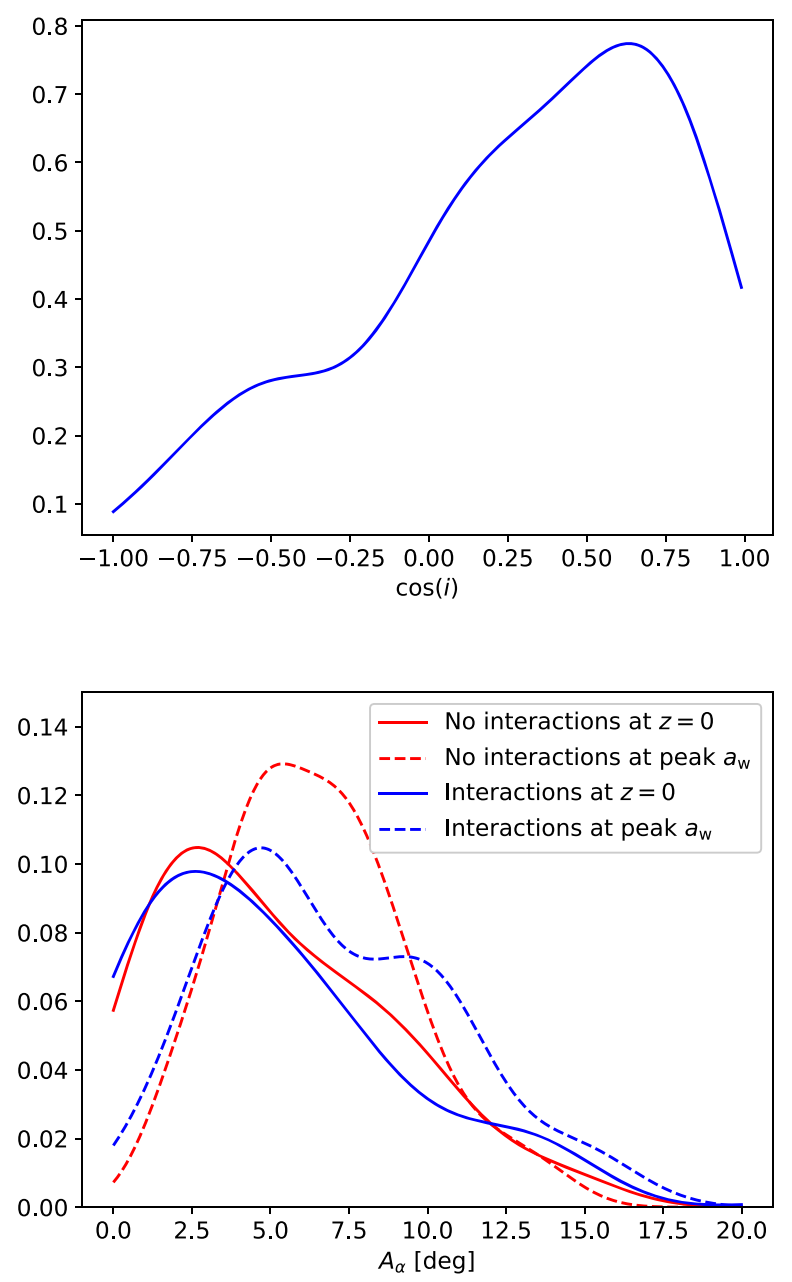

Figure 9. Upper panel: Normalized histogram of the cosine of the incident angle $i$ for galaxies that were warped by the interactions (blue line). Lower panel: Normalized histograms of the asymmetry parameter $A_{\alpha}$ measured for the sample of interacting galaxies at $z=0$ (blue solid line) and at the time when $a_{\mathrm{w}}$ peaks, i.e. shortly after the pericentre passage of the perturber (blue dashed line) compared with the histogram for all other S-shaped warps that were not interacting, measured at $z=0$ (red solid line) and at the times of theirs peak of $a_{\mathrm{w}}$ (red dashed line).

interpretation of this finding was that these angles optimize the combined effect of two factors: the time-scale of the interaction, which is greatest for $i=0^{\circ}$ and $180^{\circ}$, and the strength of the vertical component of the tidal force, which is greatest for $i=90^{\circ}$ and $270^{\circ}$. For the tidal warp formation, both of these factors contribute. We agree with the interpretation of Kim et al. (2014) and our results partly confirm their findings, with an important modification that cases closer to prograde are more effective in warp formation in agreement with the interpretation based on resonant behaviour (D'Onghia et al. 2010; Łokas et al. 2015).

\subsection{Asymmetry of tidally induced warps}

García-Ruiz et al. (2002) and Ann \& Park (2006) found that warps of galaxies that are interacting are more asymmetrical, i.e. one side of them is longer than the other one. To verify whether this trend is also present in IllustrisTNG warps, we define the asymmetry parameter

$A_{\alpha}=\left|\arctan \left(\left|a_{\text {up }}\right|\right)-\arctan \left(\left|a_{\text {down }}\right|\right)\right|$, where $a_{\text {up }}$ and $a_{\text {down }}$ are values of coefficients $a_{\mathrm{w}}$ for the two highest values corresponding to the two tips of the warp (one pointing up and the other down). This parameter is inspired by the asymmetry parameter from equation (4) of Ann \& Park (2006); however, in our case, $\arctan$ of $a_{\text {up }}$ and $a_{\text {down }}$ are not exactly the warp angles $\alpha$ defined as the inclinations of lines coming from the centre of the galaxy to the edges of the warp (see fig. 2 of Kim et al. 2014). In Section 3.1, we showed that our parametrization of warps proved to be a good proxy for the deviation of warps from the equatorial planes of discs and it is very useful for a large sample of investigated galaxies.

The lower panel of Fig. 9 shows normalized histograms for $A_{\alpha}$ measured for our sample of 66 galaxies that had their warps induced by interactions and for the remaining 121 warped galaxies for which we did not find any connection between the warps and interactions. For both samples, we measured the asymmetry at $z=0$ and at the time where $a_{\mathrm{w}}$ was peaking, which for the warps from interactions was shortly after the pericentre passage of the perturber that induced warps and for the other sample it was just the last peak that is probably associated with some other source of warping that happened just before that. For both samples, the asymmetry is higher shortly after the warps were induced in comparison to $z=0$. It means that whatever the source of the warping was (interactions or some other processes), initially the distortion is more pronounced on one side than the other; however, with time it decreases and warps can be better characterized as self-consistent, rather than driven. Warps from interactions are slightly more asymmetric that the other sample; however, the difference is very small (mean asymmetry for warps from interactions at peak time is $7^{\circ}$ and for warps not from interactions is $6.5^{\circ}$ ). We interpret these results with comparison to observations that find that interacting warps are more asymmetric as follows. All warps are asymmetric at the beginning of their lifetime and the asymmetry decreases with time. In observations, those that are associated with interactions have higher asymmetries, because for them to be classified as interacting, the perturber galaxy has to be in proximity, which means that they are always observed at the specific time in their evolution, shortly after the triggering. Meanwhile, all other warps are observed at a random time of their evolution (without the constrain to have a perturber nearby) and on average their asymmetry falls lower.

\subsection{Warps from the gas accreted by satellites}

In Section 3.2, we interpreted the first two examples from Fig. 5 as having their S-shaped warps induced not only by the tidal interaction but also by the satellite accreting gas on to the disc of the host. This interpretation, however, was proposed based only on visual inspection of the maps like those in Fig. 5, where some clouds of gas seem to be following the satellite and later the warp appears. This interpretation could be wrong and only result from a misleading visual impression. To tell whether the satellites are responsible for the gas accretion that enhances warps, one needs to track positions of individual gas cells from snapshot to snapshot. This task is particularly tricky and computationally expensive when using the IllustrisTNG data, where gas cells are continually changing their identity and turning into stars. To track the gas, it is necessary to use tracer particles, which are not catalogued into any subgroups, so the loop over all tracers in the snapshot has to be done to find the interesting subset. Tracking the gas is also difficult specifically in the TNG100 simulation, because tracers are saved only in so-called full snapshots, which are fewer and saved less frequently than regular snapshots. 
For the reasons described above, we chose to study only one example that we suspected to have its warp significantly enhanced by gas accretion. Using maps similar to those in Fig. 5, we chose this particular case after seeing a lot of movement in the surrounding gas. Also, the fact that the rise of the warp occurred between two full snapshots was a factor that made this case interesting and feasible to study.

This example is presented in Fig. 10, where the two top plots show the column density of the gas of the host, with gas cells of the perturber overlapped in orange. These two plots were made using two full snapshots before (i.e. at a time marked as A in Fig. 10) and after (i.e. at a time marked as B in Fig. 10) the pericentre passage of the satellite and the time of the rise of the warp (see Fig. 11). However, the gas cells of the perturber were taken one full snapshot earlier at 10.26 Gyr to ensure greater separation between the two galaxies. It is clear that the majority of gas cells of the perturber were correctly assigned to it before the pericentre (the incorrect assignment would mean that some gas cells from the halo would be associated with the perturber, and while the galaxy would move, these cells would remain in the halo instead of following the perturber) and later they end up being accreted on the host and contributing to the warp. We found that between the times $\mathrm{A}$ and $\mathrm{B}$ the mass of the gas within the cylinder of the host limited by $|z|<2 R_{1 / 2 *}$ and $R<5 R_{1 / 2 *}$ (see Section 3.1) increased by around 50 percent. The third plot shows the host's gas distribution without the contribution from the satellite, to discriminate which parts of the warp the perturber is contributing the most. After the time marked as B, the satellite is quickly accreted and absorbed by the host (see Fig. 11), which guarantees that the gas that contributes to the warp at B does not escape anywhere, which could be possible if, for example, the satellite was leaving the host with a very high velocity. Fig. 11 also shows that previous passages of this satellite induced the warp in the past and it is a recurrent event. However, we did not check whether the previous passages also accreted gas (surely this accretion could not be as strong as between times $\mathrm{A}$ and $\mathrm{B}$ because the previous pericentre was $\sim 3$ times larger).

Due to technical limitations described at the beginning of this section, we only checked for this one case how the gas accreted by the satellite can influence the warp. We found here that it is an important factor and an alternative driving force of the warping, together with tidal interactions. This scenario can be further investigated in zoom-in or idealized simulations to verify its importance (e.g. two simulations differing only in the gas content of the satellite can show how much stronger the resulting warp is in the case with the gas accretion).

\section{DISCUSSION AND SUMMARY}

\subsection{Comparison with previous studies}

Several studies were already performed aiming to investigate tidally induced warps; however, it is difficult to compare our results with these findings, as they focus mostly on stellar warps. On the other hand, studies that focus on disturbed gaseous discs did not explore in great detail interactions as a possible origin of vertical morphologies, and therefore we can only compare a few general results with them. For example, Bahé et al. (2016) classified by eye $2200 \mathrm{H}$ I gas discs from the EAGLE simulations and found that around two-thirds of them are disturbed. This is in a rough agreement with our findings that around half of gas discs are not flat and the discrepancy in value may arise from various effects (e.g. different simulation methods or human bias in classification).

Gómez et al. (2017) investigated warps and corrugations in stellar discs of 16 MW-like analogues in a cosmological zoom-in Auriga
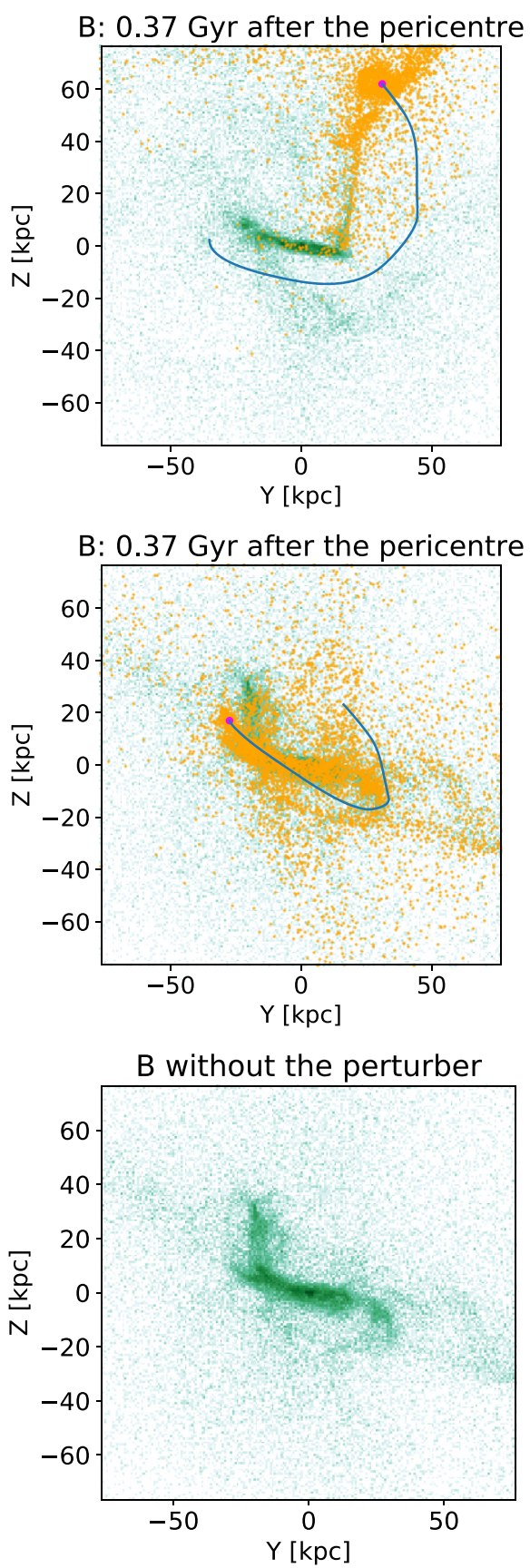

Figure 10. Upper panel: Column density of gas of the host before the pericentre passage (colour map) with gas cells assigned to the perturber (orange points). Blue line is the orbit of the perturber and the magenta dot is its centre. Middle panel: Same as the first, but after the pericentre passage. Lower panel: Same as the second, but without the tracer gas cells of the perturber. Subhalo ID of the host in this case at $z=0$ is 494091. $Y$-axis is not the same in the top and middle and lower panels since galaxies were levelled at every snapshot and the projections changed.

simulation. They found that the majority of their warped stellar discs had a significant past tidal interaction, which agrees with our conclusions that interactions play an important role in warp formation. They determined the minimal mass of a perturber to influence an MW-sized galaxy to be $\sim 10^{10} \mathrm{M}_{\odot}$, which by assuming the mass of the MW around $\sim 10^{12} \mathrm{M}_{\odot}$ gives a rough fraction of 


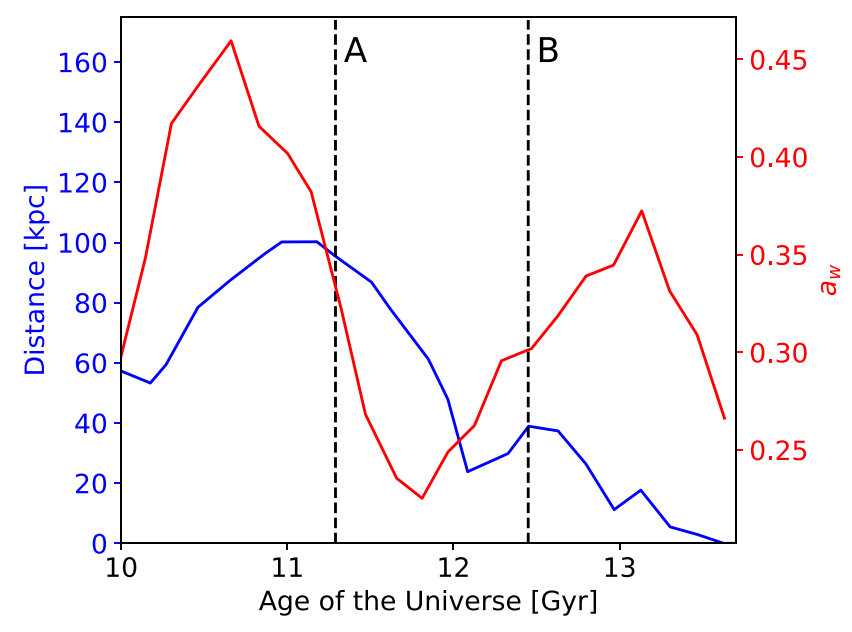

Figure 11. Same as Figs 6 and 7, but for the case discussed in Section 3.6 with marked times A (before the pericentre) and B (after the pericentre), which are shown in Fig. 10.

1 percent of the host's mass. This is consistent with Fig. 8, where almost no cases were found for mass ratios smaller than 1 percent. Gómez et al. (2017) also found that, for most of their cases, stellar warps were followed by the star-forming gaseous disc. We checked whether stellar counterparts of our interaction-driven warps show similar structures at $z=0$ and found that 20 out of 66 stellar discs show similar warps but with smaller amplitudes than gaseous ones, which is to some extent expected from observations. The remaining 46 stellar discs seemed flat at $z=0$ probably because the last pericentre in those cases was on average $>0.8$ Gyr earlier than for the cases with stellar warps.

Kim et al. (2014) performed idealized $N$-body simulations to study tidally induced stellar warps. Despite the difference in the disc component that we were interested in, some of our conclusions seem to be consistent. In both Kim et al. (2014) and our study, the preferred values of the incident angles were found to lie around $45^{\circ}$ and $135^{\circ}$ and tidally induced warps were asymmetric with asymmetries not exceeding the values of $6^{\circ}$ in their case and $15-16^{\circ}$ in ours. These agreements and the results of their only simulation with gas (presented in their fig. 11) suggest that warps induced by interactions behave similarly in gas and stars. The only difference seems to be the amplitude, which we found to be higher in gas discs, and this also seems to agree with their fig. 11. This happens probably because gas discs are more extended and less bound in the outer parts and therefore more sensitive to perturbations. Discrepancies between the particular numerical values of lifetimes of warps obtained in this study and the work of Kim et al. (2014) can be attributed to many differences among which the most important seem to be the fact of studying different components, different simulation set-ups and methods, and different ways of measuring warp parameters.

\subsection{Summary}

Observational correlations and results of numerical simulations have already demonstrated the importance of tidal interactions for warp formation in spiral galaxies. In this paper, we used the TNG100 simulation from the IllustrisTNG set to investigate for the first time the importance of tidal interactions for the formation of S-shaped gas warps in galaxies in a cosmological context. Using a sample of 1593 spiral galaxies that had sufficient resolution and gas content to reproduce gas warps, we found that 15.6 percent of them had specific S-shaped warps. The S-shaped and other vertical distortions were characteristic of 46.3 per cent of all galaxies from this sample, which agrees with observational findings on the frequency of warps (Bosma 1991; García-Ruiz et al. 2002).

We found that around one-third of the sample of S-shaped warped galaxies was induced by interactions with other galaxies. Half of these interactions ended in the perturber being absorbed by its host, while in the other half perturbers maintained their identity at the end of the simulation. Gas warps induced by interactions in our sample tend to have a lifetime of $<1 \mathrm{Gyr}$; however, recurrent passages of satellites can regenerate them. We found that all warps, including those driven by interactions, are more asymmetric early in their lifetimes and with time they settle into more symmetric configurations. Consistently with simulations of Kim et al. (2014), we found that angles between the angular momentum of the host and the orbital angular momentum of the perturber that preferentially lead to warp formation are around $40-50^{\circ}$ and to a lesser extent $120-130^{\circ}$. These values are optimal to balance the time of the interaction and the vertical component of the tidal force, which are both important for warp formation. Finally, we found that accretion of the gas from perturbers also contributes to the formation of warps. However, the structure and time resolution of the simulations did not allow us to investigate in greater detail how strong this effect is and in how many cases it is comparable to the tidal evolution.

In future work, we plan to investigate the origin of the remaining two-thirds of the S-shaped warps found in this study, to understand better the mechanisms of warp formation.

\section{ACKNOWLEDGEMENTS}

This work was supported in part by the National Science Centre, Poland under grant 2013/10/A/ST9/00023 and by the STFC grant $\mathrm{ST} / \mathrm{S} 000453 / 1$. We are grateful to an anonymous referee for useful comments that helped to improve the paper. We are thankful to B.-E. Semczuk for the contribution to the visual classification. We appreciate insightful discussions with I. Ebrova, N. Peschken, D. Nelson, and A. Pillepich that contributed to this paper. EDO acknowledges support from the Center for Computational Astrophysics at Flatiron Institute for the hospitality during the completion of this work. VPD was supported by STFC consolidated grant ST/R000786/1. The simulation TNG100 that was used in this work is the flagship run of the IllustrisTNG project and it was run on the HazelHen Cray XC40 system at the High Performance Computing Center Stuttgart as part of the project GCS-ILLU of the Gauss Centre for Supercomputing. We are thankful to the IllustrisTNG team for granting us early access to the simulation data.

\section{DATA AVAILABILITY}

The main data body that was used in the paper, which is the IllustrisTNG simulation, is publicly available at https://www.tngproject.org/. The processed data that are the result of the analysis presented in the paper can be shared on reasonable request to the corresponding author.

\section{REFERENCES}

Ann H. B., Park J. C., 2006, New Astron., 11, 293

Bahé Y. M. et al., 2016, MNRAS, 456, 1115

Battaner E., Jimenez-Vicente J., 1998, A\&A, 332, 809

Battaner E., Florido E., Sanchez-Saavedra M. L., 1990, A\&A, 236, 1 
Blumenthal K. et al., 2020, MNRAS, 492, 2075

Bosma A., 1991, in Casertano S., Sackett P. D., Briggs F. H., eds, Warped Disks and Inclined Rings Around Galaxies. Cambridge Univ. Press, Cambridge, p. 181

Briggs F. H., 1990, ApJ, 352, 15

Burke B. F., 1957, AJ, 62, 90

Byrd G. G., Howard S., 1992, AJ, 103, 1089

Chen X., Wang S., Deng L., de Grijs R., Liu C., Tian H., 2019, Nat. Astron., 3,320

Debattista V. P., Sellwood J. A., 1999, ApJ, 513, L107

D’Onghia E., Vogelsberger M., Faucher-Giguere C.-A., Hernquist L., 2010, ApJ, 725, 353

D’Onghia E., Madau P., Vera-Ciro C., Quillen A., Hernquist L., 2016, ApJ, 823,4

Faucher-Giguère C.-A., Lidz A., Zaldarriaga M., Hernquist L., 2009, ApJ, 703,1416

García-Ruiz I., Sancisi R., Kuijken K., 2002, A\&A, 394, 769

Genel S. et al., 2014, MNRAS, 445, 175

Gómez F. A., White S. D. M., Marinacci F., Slater C. T., Grand R. J. J., Springel V., Pakmor R., 2016, MNRAS, 456, 2779

Gómez F. A., White S. D. M., Grand R. J. J., Marinacci F., Springel V., Pakmor R., 2017, MNRAS, 465, 3446

Kerr F. J., 1957, AJ, 62, 93

Kim J. H., Peirani S., Kim S., Ann H. B., An S.-H., Yoon S.-J., 2014, ApJ, 789, 90

Kollatschny W., Dietrich M., 1990, A\&A, 233, 333

Kuijken K., Garcia-Ruiz I., 2001, in Funes J. G., Corsini E. M., eds, ASP Conf. Ser. Vol. 230, Galaxy Disks and Disk Galaxies. Astron. Soc. Pac., San Francisco, p. 401

Łokas E. L., Semczuk M., Gajda G., D’Onghia E., 2015, ApJ, 810, 100

Marinacci F. et al., 2018, MNRAS, 480, 5113

Masset F., Tagger M., 1997, A\&A, 318, 747

Naiman J. P. et al., 2018, MNRAS, 477, 1206

Nelson D. et al., 2018, MNRAS, 475, 624

Nelson D. et al., 2019a, Comput. Astrophys. Cosmol., 6, 2

Nelson D. et al., 2019b, MNRAS, 490, 3234

Newton K., Emerson D. T., 1977, MNRAS, 181, 573

Oh S. H., Kim W.-T., Lee H. M., 2015, ApJ, 807, 73

Pakmor R., Springel V., 2013, MNRAS, 432, 176

Peschken N., Łokas E. L., 2019, MNRAS, 483, 2721

Pillepich A. et al., 2018a, MNRAS, 473, 4077

Pillepich A. et al., 2018b, MNRAS, 475, 648

Pillepich A. et al., 2019, MNRAS, 490, 3196

Planck Collaboration XIII, 2016, A\&A, 594, A13

Poggio E. et al., 2018, MNRAS, 481, L21

Poggio E., Drimmel R., Andrae R., Bailer-Jones C. A. L., Fouesneau M., Lattanzi M. G., Smart R. L., Spagna A., 2020, Nat. Astron., 4, 590

Pop A.-R., Pillepich A., Amorisco N., Hernquist L., 2017, Galaxies, 5, 34

Pop A.-R., Pillepich A., Amorisco N. C., Hernquist L., 2018, MNRAS, 480, 1715

Rahmati A., Pawlik A. H., Raičević M., Schaye J., 2013, MNRAS, 430, 2427

Reshetnikov V., Combes F., 1998, A\&A, 337, 9

Rodriguez-Gomez V. et al., 2019, MNRAS, 483, 4140

Rogstad D. H., Wright M. C. H., Lockhart I. A., 1976, ApJ, 204, 703

Romero-Gómez M., Mateu C., Aguilar L., Figueras F., Castro-Ginard A., 2019, A\&A, 627, A150

Rosas-Guevara Y. et al., 2020, MNRAS, 491, 2547

Roškar R., Debattista V. P., Brooks A. M., Quinn T. R., Brook C. B., Governato F., Dalcanton J. J., Wadsley J., 2010, MNRAS, 408, 783

Schönrich R., Dehnen W., 2018, MNRAS, 478, 3809

Schwarzkopf U., Dettmar R. J., 2001, A\&A, 373, 402

Semczuk M., Łokas E. L., Salomon J.-B., Athanassoula E., D’Onghia E., 2018, ApJ, 864, 34

Shen J., Sellwood J. A., 2006, MNRAS, 370, 2

Skowron D. M. et al., 2019, Science, 365, 478

Springel V., 2010, MNRAS, 401, 791

Springel V., Hernquist L., 2003, MNRAS, 339, 289

Springel V. et al., 2018, MNRAS, 475, 676
Vesperini E., Weinberg M. D., 2000, ApJ, 534, 598

Vogelsberger M. et al., 2014a, MNRAS, 444, 1518

Vogelsberger M. et al., 2014b, Nature, 509, 177

Weinberger R. et al., 2017, MNRAS, 465, 3291

Yun K. et al., 2019, MNRAS, 483, 1042

Zhu Q. et al., 2018, MNRAS, 480, L18

\section{APPENDIX A: MORE DETAILED WARP FITS}

In Section 3.1, we applied simple straight-line fits to cylindrical slices of the gas cells in order to describe warp inclinations. Previous studies, such as Chen et al. (2019) or Skowron et al. (2019), used more detailed fits to describe the geometry of the MW's stellar warp. Their fits can be summarized as

$$
\begin{array}{ll}
z(R)=0, & \text { for } R<R_{\mathrm{W}}, \\
z(R)=a_{\mathrm{w}}\left(R-R_{\mathrm{W}}\right)^{b} \sin \left(\phi-\phi_{\mathrm{W}}\right), & \text { for } R>R_{\mathrm{W}} .
\end{array}
$$

We adopted this two-component approach (with setting $b \equiv 1$ ) for our samples of S-shaped warps and flat discs in order to test whether this more accurate method is suitable for data coming from cosmological simulations.

We found that for some cases equation (A1) indeed is more accurate to describe warp geometry (e.g. upper panel of Fig. A1) as found in studies about MW. Nevertheless, for many cases in the studied simulation the surrounding gas cells that are present in the gas halo can drastically alter these fits and bias the results. This is clearly visible in the lower panel of Fig. A1, where a relatively flat gas disc yields a high value of $a_{\mathrm{w}}$, due to gas cells present above the disc. These cells were also present in the method discussed in Section 3.1; however, there they were not as influential, because fitting equation (2) to the whole range of radii assured that numerous
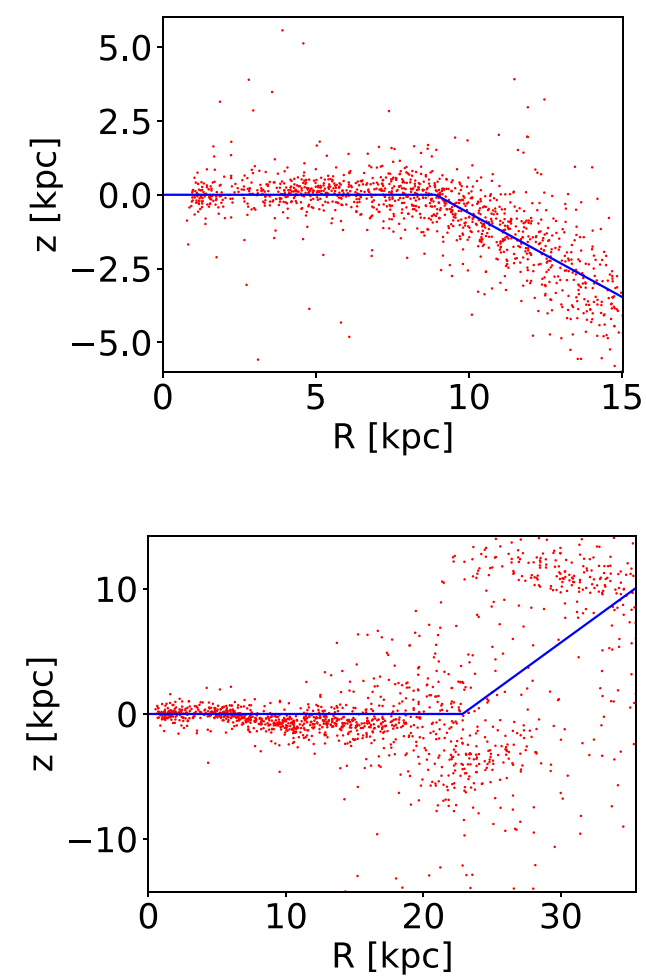

Figure A1. Upper panel: An example fit of equation (A1) that describes warp's geometry well. Lower panel: An example fit of the same equation that yields false result; i.e. gas clouds above the flat discs increase the value of $a_{\mathrm{w}}$. 


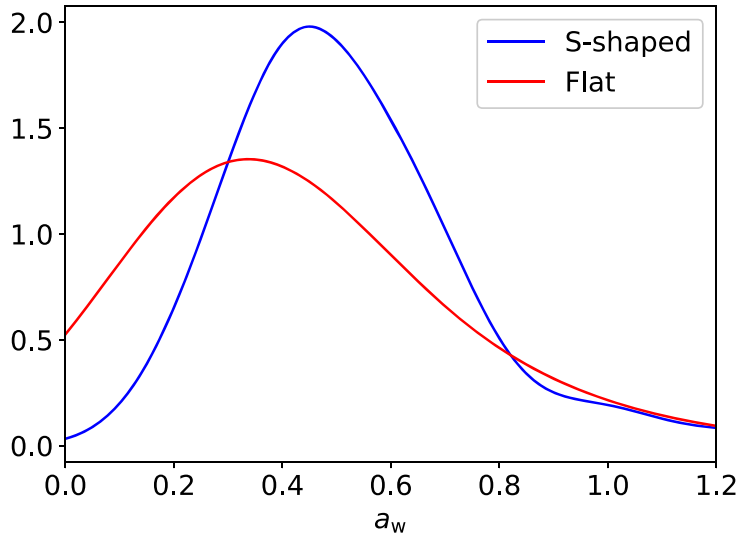

Figure A2. Same as Fig. 3, but for the fitting procedure described in the appendix. inner gas disc cells would outweigh the clouds in the halo and for the flat discs $a_{\mathrm{w}}$ would remain relatively low.

For reasons discussed above, we decided to keep the simple fitting procedure as in Section 3.1, because the error made that way (i.e. fitting a straight line to warps that are better described with a break) is smaller than the error of the method discussed in the appendix. The whole procedure was constructed to tell when the flat disc became warped and the straight-line fit worked better for this purpose. This is clear when comparing Fig. 3 with Fig. A2, which shows a significant overlap in $a_{\mathrm{w}}$ between visually classified flat and S-shaped warped discs. The method introduced in the appendix would require a cleaning algorithm that would select only disc cells for the fitting procedure and while working on this project we could not find an algorithm precise enough to do so.

This paper has been typeset from a $\mathrm{T}_{\mathrm{E}} \mathrm{X} / \mathrm{L} \mathrm{T}_{\mathrm{E}} \mathrm{X}$ file prepared by the author. 